\title{
Computation of Opacities for Diatomic Molecules
}

\author{
Robert L. Kurucz \\ Harvard-Smithsonian Center for Astrophysics, 60 Garden St., Cambridge, MA 02138, \\ USA
}

\section{Atomic and Molecular Data}

In this section I briefly describe my efforts to improve the atomic and molecular line data. This work is described in more detail in Kurucz (1992a). In subsequent sections I briefly describe three methods for computing opacity and the models and spectra that result from using them.

My model calculations in the 1970 s used the distribution-function line opacity computed by Kurucz $(1979 a, b)$ from the line data of Kurucz \& Peytremann (1975). We had computed $g f$ values for 1.7 million atomic lines for sequences up through nickel using scaled-Thomas-Fermi-Dirac wavefunctions and eigenvectors determined from least squares Slater parameter fits to the observed energy levels. We also collected all published data on $g f$ values and included them in the line list whenever they appeared to be more reliable than the computed data (that work is ongoing, but I am running behind).

After the Kurucz-Peytremann calculations were published, I started work on line lists for diatomic molecules beginning with $\mathrm{H}_{2}, \mathrm{CO}$, and $\mathrm{SiO}$. Next, Lucio Rossi of the Istituto Astrofisica Spaziale in Frascati, John Dragon of Los Alamos, and I computed line lists for electronic transitions of $\mathrm{CH}, \mathrm{NH}, \mathrm{OH}, \mathrm{MgH}, \mathrm{SiH}$, $\mathrm{CN}, \mathrm{C}_{2}$, and $\mathrm{TiO}$. In addition to lines between known levels, these lists include lines whose wavelengths are predicted and are not good enough for detailed spectrum comparisons but are quite adequate for statistical opacities. All these data are listed in Table 1.

In 1983 I recomputed the opacities using the additional atomic and molecular data described above which totalled 17,000,000 lines. These opacities were used to produce improved empirical solar models (Avrett et al. 1984), but were found to still not have enough lines. In detailed ultraviolet spectrum calculations, half the intermediate strength and weak lines were missing. This discrepancy was caused by missing iron group atomic lines that go to excited configurations that had not yet been observed in the laboratory and so had not been included in the Kurucz-Peytremann calculations.

I was granted a large amount of computer time at the San Diego Supercomputer Center to carry out new calculations. I included as many configurations for 


\section{Table 1. Molecular Line Lists}

\begin{tabular}{|c|c|c|c|c|}
\hline \multirow[t]{2}{*}{ FILE } & \multirow{2}{*}{$\begin{array}{l}\text { NUMBER } \\
\text { OF LINES }\end{array}$} & \multicolumn{2}{|c|}{ WAVELENGTH (NM) } & \multirow[t]{2}{*}{ COMMENT } \\
\hline & & FIRST & LAST & \\
\hline $\mathrm{H} 2$ & 28486 & 84.4941 & 184.4573 & Lyman and Werner \\
\hline HYDRIDES & 331268 & 203.6264 & 3245.1715 & $\mathrm{CH}, \mathrm{NH}, \mathrm{OH}, \mathrm{MgH}, \mathrm{SiH}$ all isotopes \\
\hline COAX & 396004 & 111.3365 & 460.6524 & CO 4th Pos all isotopes \\
\hline COIR & 118920 & 963.5078 & 9998.8853 & CO vib-rot all isotopes \\
\hline SIOAX & 760378 & 177.3128 & 546.2833 & $\mathrm{SiO} \mathrm{A}-\mathrm{X}$ all isotopes \\
\hline SIOEX & 947015 & 143.0468 & 462.4214 & SiO $\mathrm{E}-\mathrm{X}$ all isotopes \\
\hline CNAX12 & 484709 & 292.5406 & 99912.800 & ${ }^{12} \mathrm{C}^{14} \mathrm{~N}$ Red \\
\hline CNAX13 & $\mathbf{5 0 3 6 3 1}$ & 295.1871 & 99924.784 & ${ }^{4} \mathrm{~N}$ Red \\
\hline CNAX15 & 289887 & 341.5994 & 99776.188 & ${ }^{5} \mathrm{~N}$ Red \\
\hline CNBX & 323818 & 201.9947 & 715.7552 & CN Violet all isotopes \\
\hline $\mathrm{C} 2 \mathrm{AX}$ & 406236 & 271.8071 & 9999.6289 & $\mathrm{C}_{2}$ Phillips all isotopes \\
\hline C2BA12 & 462803 & 389.6061 & 99957.754 & Ballik-Ramsay \\
\hline C2BA13 & 387809 & 511.6587 & 99980.746 & Ballik-Ramsay \\
\hline С2BA33 & 313319 & 527.1495 & 80319.395 & C Ballik-Ramsay \\
\hline C2DA 12 & 329771 & 344.9621 & 2541.6874 & ${ }^{12} \mathrm{C}^{12} \mathrm{C}$ Swan \\
\hline C2DA13 & 253014 & 374.0201 & 991.5625 & ${ }_{3} \mathrm{C}$ Swan \\
\hline C2DA33 & 217545 & 376.2757 & 959.4157 & C Swan \\
\hline $\mathrm{C} 2 \mathrm{EA} 12$ & 487232 & 176.0052 & 862.4585 & C Fox-Herzberg \\
\hline C2EA13 & 332214 & 177.3769 & 560.9010 & C Fox-Herzberg \\
\hline C2EA33 & 260883 & 178.6938 & 543.2282 & C Fox-Herzberg \\
\hline TIOAX6 & 325116 & 528.5640 & 1875.4212 & ${ }^{16} \mathrm{O} \gamma$ \\
\hline TIOAX7 & 327496 & 528.7059 & 1771.6149 & ${ }^{16} \mathrm{o} \gamma$ \\
\hline TIOAX8 & 647203 & 490.4263 & 2146.0045 & ${ }^{48} \mathrm{Ti}$ \\
\hline TIOAX9 & 332113 & 528.9757 & 1864.4024 & ${ }^{49} \mathrm{Ti}$ \\
\hline TIOAXO & 334222 & $\mathbf{5 2 9 . 1 0 4 6}$ & 1860.9887 & ${ }^{50} \mathrm{Ti}^{16} \mathrm{O} \gamma$ \\
\hline TIOBX6 & 343598 & 246.6118 & 1232.9218 & ${ }^{46} \mathrm{Ti}^{16} \mathrm{O} \gamma^{\prime}$ \\
\hline TIOBX7 & 345990 & 246.3570 & 1209.4093 & ${ }^{47} \mathrm{Ti}^{16} \mathrm{O} \gamma^{\prime}$ \\
\hline ТІОВX8 & 861488 & 235.9828 & 1362.8784 & ${ }^{48} \mathrm{Ti}^{16} \mathrm{O} \gamma^{\prime}$ \\
\hline TIOBX9 & 350530 & 246.7795 & 1187.2650 & ${ }^{49} \mathrm{Ti}^{16} \mathrm{O} \gamma^{\prime}$ \\
\hline TIOBXO & 352802 & 247.3491 & 1186.9649 & ${ }^{50} \mathrm{Ti}^{16} \mathrm{O} \gamma^{\prime}$ \\
\hline TIOCX6 & 232718 & 350.0947 & 1562.0368 & ${ }^{46} \mathrm{Ti}^{16} \mathrm{O} \alpha$ \\
\hline TIOCX7 & 234318 & 350.0471 & 1554.5985 & ${ }^{47} \mathrm{Ti}^{16} \mathrm{O} \alpha$ \\
\hline TIOCX8 & 503950 & 257.0800 & 1316.1735 & ${ }_{49}^{48} \mathrm{Ti}^{16} \mathrm{O} \alpha$ \\
\hline TIOCX9 & 237430 & 350.0113 & 1540.7715 & ${ }^{49} \mathrm{Ti}^{10} \mathrm{O} \alpha$ \\
\hline TIOCXO & 238827 & 349.9744 & 1534.3348 & ${ }^{00} \mathrm{Ti}^{10} \mathrm{O} \alpha$ \\
\hline TIOEX6 & 241398 & 630.6768 & 2002.6215 & $47 \mathrm{Ti}$ O \\
\hline TIOEX7 & 242954 & 631.0335 & 1997.7022 & $48_{\mathrm{T}}{ }^{16} \mathrm{OC}$ \\
\hline TIOEX8 & 510074 & 549.4411 & 2273.1493 & ${ }_{49} \mathrm{Ti}_{16} \mathrm{OE}$ \\
\hline TIOEX9 & 245978 & $\begin{array}{l}631.7067 \\
632.0252\end{array}$ & $\begin{array}{l}1988.4477 \\
1984.0965\end{array}$ & ${ }^{50} \mathrm{Ti}^{16} \mathrm{OE}$ \\
\hline TIOEXO & $\begin{array}{l}247466 \\
310376\end{array}$ & $\begin{array}{l}632.0252 \\
541.4458\end{array}$ & $\begin{array}{l}1984.0965 \\
2250.2486\end{array}$ & TiO $\delta$ all isotopes \\
\hline $\begin{array}{l}\text { TIOBA } \\
\text { TIOCA }\end{array}$ & $\begin{array}{l}310376 \\
223850\end{array}$ & $\begin{array}{l}541.4458 \\
446.7588\end{array}$ & $\begin{array}{l}2250.2486 \\
1669.0456\end{array}$ & TiO $\beta$ all isotopes \\
\hline $\begin{array}{l}\text { TIOCA } \\
\text { TIOFA }\end{array}$ & $\begin{array}{l}223850 \\
153496\end{array}$ & 432.0083 & 816.6252 & TiO f-a all isotopes \\
\hline TIOBD & 158000 & 593.4372 & 2662.9648 & TiO $\phi$ all isotopes \\
\hline TIOED & 102107 & 332.9690 & 562.5112 & TiO e-d all isotopes \\
\hline
\end{tabular}


each ion as I could fit into a Cray. Hamilitonian parameters were determined by combining least squares fits for levels that have been observed with computed Hartree-Fock integrals (scaled) for higher configurations. All configuration interactions were included. My computer programs have evolved from Cowan's (1968) programs. Transition integrals were computed with scaled-Thomas-Fermi-Dirac wavefunctions and the whole transition array was produced for each ion. Radiative, Stark, and van der Waals damping constants and Landé g values are automatically produced for each line. The first nine ions of $\mathrm{Ca}$ through $\mathrm{Ni}$ produced 42,000,000 lines. Most of those lines have uncertain wavelengths because they go to predicted rather than measured levels. I have produced a single tape edition of these data for distribution that has all the lines with reliable wavelengths between laboratory determined energy levels.

I hope to spend the next year improving the line data. I will extend the atomic calculation to elements lighter and heavier than the iron group which I have already computed. I will recompute the energy levels and line lists whenever new laboratory analyses become available and I will make the predictions available to laboratory spectroscopists. Several of the iron group calculations have already been revised. Because computers are now more powerful, more configurations can be treated. This should account for more of the missing infrared lines because they are usually transitions between highly excited levels. I will also try to include as much hyperfine and isotopic splitting data as possible (Kurucz 1993b).

Returning to the diatomic molecules, the line lists in Table 1 are in need of revision. All the transitions are electronic except for the $\mathrm{CO}$ vibration-rotation bands. Most of the isotopic positions are unreliable. Most of the calculations were done more than 15 years ago. I plan to include all the improvements in the laboratory analyses since that time and I plan to add all the significant vibrationrotation bands and many ultraviolet electronic bands. The newer analyses are based on FTS spectra and produce dramatic improvements in energy levels and line positions. However, they still do not go to high enough $\mathrm{V}$ and $\mathrm{J}$. Farrenq et al. (1990) have actually been able to use the solar spectrum itself to analyse CO to high J (see also Grevesse \& Sauval this volume).

The procedure I use for generating line lists is straightforward and produces all the lines up to a specified cut-off lower energy level. I start with all the known energy levels. I set up a model rotational Hamiltonian and then I do a least squares fit to determine the rotational constants. Once the fit has converged, I use the Hamiltonian to generate all possible eigenvalues and eigenvectors. The eigenvalues are replaced by the observed energies where they are known. I compute the RKR potential and then all the vibrational wavefunctions. I then integrate over measured or computed transition moments taken from the literature to get the transition integrals. The transition integrals are divided into transition arrays in the adopted basis and are transformed to observed coupling using the eigenvectors. Given enough computer time, I can readily generate thousands of energy levels and millions of lines. Since the known energy levels are used when available, the line wavelengths are correct for lines between known energy levels, regardless of perturbations. Lines to predicted levels are as accurate as the 
least squares fitting procedure. Radiative damping constants are automatically produced for each line.

Line lists for the triatomic molecules are needed for work on cool M stars. I hope to obtain them from Jørgensen \& Jensen (1993) and other groups.

All the atomic and molecular data will be published on CD-ROMs. These will include both a replacement for Kurucz-Peytremann (1975) and all lines with good wavelengths for molecules listed in Table 1 for use in spectrum synthesis calculations. Highly compressed versions of the atomic and diatomic data have already been published for use in opacity calculations as indicated in Table 2 . This allows anyone to compute opacities or opacity-sampled model atmospheres for arbitrary abundances. For each major atomic or molecular calculation I expect to publish a CD-ROM on which I will provide tables of all laboratory measurements, together with my computed energy levels, eigenvector compositions, damping constants, Landé $g$ values, lifetimes, branching ratios, and $g f$ values. These should be of value to both laboratory and astronomical spectroscopists.

\section{The Distribution Function Opacity Program}

The opacities are calculated with a version of my spectrum synthesis computer program that has been under development since 1965 and has been described by Kurucz \& Avrett (1981). The algorithms for computing the total line opacity are extremely fast because maximum use is made of temperature and wavelength factorization and pretabulation. Up to a million point spectrum can be computed in one run. There is no limit to the number of spectrum lines that can be treated.

The spectrum calculations require a pre-existing model atmosphere. For computing opacities it is just a table of temperatures and pressures. Quantities that need be computed only once for the model atmosphere are pretabulated, i.e., number densities, partition functions, and doppler widths. The equation of state and continuum opacities are computed with a version of the model atmosphere program ATLAS (Kurucz 1970). Solar abundances are taken from Anders \& Grevesse (1989). Photoionization continua are put in at their exact positions, each with its own cross-section and with the series of lines that merge into each continuum included so that there are no discontinuities in the spectrum.

Hydrogen line profiles are computed using a routine from Peterson (1979) that approximates the Vidal et al. (1973) profiles, works to high $\mathrm{n}$, and includes Doppler broadening, resonance broadening, van der Waals broadening, and fine-structure splitting. Autoionization lines have Shore-parameter Fano profiles. Other lines have Voigt profiles that are computed accurately for any value of the damping parameter $a$ which includes radiative, Stark, and van der Waals broadening. For small $a$, table lookup is used for speed. Line wings are truncated when they are weaker than 0.001 times the continuum. Lines weaker than 0.001 times the continuum are not considered.

The opacity spectrum is computed only for zero microturbulent velocity. Other velocities are obtained by convolution. The spectrum is computed at $3,500,000$ points uniformly spaced in $\Delta \lambda / \lambda$ (proportional to Doppler velocity) 
from $8.97666 \mathrm{~nm}$ to $10000 \mathrm{~nm}$. The spectral resolution of 500,000 , or $0.6 \mathrm{~km} / \mathrm{s}$, is enough to resolve line cores. The distribution function for a wavelength interval is determined by statistically analyzing the total line opacity spectrum in that interval. The intervals vary from $1 \mathrm{~nm}$ in the ultraviolet to $10 \mathrm{~nm}$ in the far infrared.

In late 1988 I used the line data described above to compute new solar abundance opacity tables for use in my modelling. The calculations involved $58,000,000$ lines, $3,500,000$ wavelength points, 56 temperatures from $2000 \mathrm{~K}$ to $200,000 \mathrm{~K}, 21 \log$ pressures from -2 to +8 , and 5 microturbulent velocities 0 , $1,2,4$, and $8 \mathrm{~km} / \mathrm{s}$, and took a large amount of computer time. The opacity was tabulated twice, as 12-step distribution functions for 328 "big" intervals for speed, and for 1212 "little" intervals for resolution.

Since the beginning of 1990 I have been able to take tremendous advantage of the new Cray YMP at the San Diego Supercomputer Center. I computed opacities ranging from 0.00001 solar to 10 times solar, enough to compute model atmospheres ranging from the oldest Population II stars to high abundance Am and Ap stars. The exact abundances are $[+1.0],[+0.5],[+0.3],[+0.2],[+0.1]$, $[+0.0],[-0.1],[-0.2],[-0.3],[-0.5],[-1.0],[-1.5],[-2.0],[-2.5],[-3.0],[-3.5],[-4.0]$, $[-4.5]$, and $[-5.0]$ where $[\mathrm{x.x}]$ stands for log difference from solar abundance of metals. I have just completed opacities with +0.4 dex enhanced $\alpha$-process elements $(\mathrm{O}, \mathrm{Ne}, \mathrm{Mg}, \mathrm{Si}, \mathrm{S}, \mathrm{Ar}, \mathrm{Ca}, \mathrm{Ti})$ for abundances [+0.5], [+0.0], [-0.5], [-1.0], $[-1.5],[-2.0],[-2.5],[-3.0],[-3.5],[-4.0],[-4.5]$, and $[-5.0]$. These opacities are distributed on CD-ROMs as listed in Table 2 or on VAX backup tapes.

Rosseland mean opacities have been computed for each abundance for use in interiors and envelope calculations.

\section{New Grids of Models}

I rewrote my model atmosphere program to use the new line opacities, additional continuous opacities, and an approximate treatment of convective overshooting. The revised version is called ATLAS9. The opacity calculation was checked by computing a realistic model for the Sun. See Kurucz (1992b) for details and figures.

Thus far, I have computed a grid of more than 7000 models for $[+1.0],[+0.5]$, $[+0.3],[+0.2],[+0.1],[+0.0],[-0.1],[-0.2],[-0.3],[-0.5],[-1.0],[-1.5],[-2.0],[-$ $2.5],[-3.0],[-3.5],[-4.0],[-4.5]$, and $[-5.0]$ for $2 \mathrm{~km} / \mathrm{s}$ microturbulent velocity. The effective temperature range is $3500 \mathrm{~K}$ to $50,000 \mathrm{~K}$, i.e. from $\mathrm{K}$ stars to $\mathrm{O}$ stars. Note that since triatomic molecules are not included in the opacity, the models cannot be used for $\mathrm{M}$ stars. Gravities range from $\log g=5$ down to 0 or the radiation pressure limit in steps of 0.5 . Models cooler than $9000 \mathrm{~K}$ are convective with $l / H=1.25$. For each model the flux was computed at 1221 wavelengths in the range 0.01 to $160 \mu \mathrm{m}$, enough to treat ionization in $\mathrm{H}$ II regions and to calibrate the infrared. The temperature range of this grid should allow photometric calibrations consistent for both cool and hot stars. 
For each model in the grid I have computed the fluxes, photometry in several systems, Balmer line profiles, and limb-darkening. The models, fluxes, colors, and Balmer line profiles are available on Kurucz CD-ROM No.13 or on VAX backup tapes. Earlier versions of these tapes had errors that have been corrected. The limb-darkening requires a separate tape for each abundance. The CD version is in preparation.

The microturbulent velocity is an important parameter that must be considered. Opacity and model structure vary with $\mathbf{v}_{\text {turb }}$. If there is diffusion, $\mathbf{v}_{\text {turb }}$ must be small. In giants it can be large. In pulsating stars, such as Cepheids and RR Lyraes, it varies with phase. I am presently extending the grid calculations to other microturbulent velocities.

I am also computing grids of models using the new $\alpha$-enhanced opacities, working inward both from the high abundance bulge stars and from the extremely low abundance halo stars.

\section{Opacity Sampling and ATLAS12}

In Am and Ap stars, or, perhaps, in all A stars, and in cool evolved giants the atmospheric abundances are not scaled-solar. Scaled-solar grids can produce only an approximate representation of the atmosphere. The cost of computing opacities is so high that it is not practical to pretabulate opacities for individual stars, although it might be possible for subclasses. The alternative is to use opacity sampling to compute individual models for each star.

I have developed a new version of my model atmosphere program, called ATLAS12, that samples from my whole line list to compute opacity. It essentially combines my spectrum synthesis program SYNTHE (Kurucz \& Avrett 1981) with my model atmosphere program ATLAS (Kurucz 1970). It recognizes more than 1000 species, each in up to 10 isotopic forms, including all ions of the elements up through $\mathrm{Zn}$ and the first 5 ions of heavier elements. The elemental abundances are treated as variable with depth.

At present ATLAS12 has 6 input files containing 58,000,000 lines:

NLTELINES: $\mathrm{H}, \mathrm{He}, \mathrm{C}, \mathrm{Mg}, \mathrm{Al}, \mathrm{Si}, \mathrm{Ca}$, etc., the file used by SYNTHE, species that can be treated in non-LTE by Avrett \& Loeser (1992);

LOWLINES: first 5 stages of ionization of all elements not in NLTELINES;

HILINES: ion stages 6 and higher of elements up through $\mathrm{Zn}$;

DIATOMICS: $\mathrm{H}, \mathrm{C}, \mathrm{N}, \mathrm{O}, \mathrm{Mg}$, $\mathrm{Si}$, etc combinations;

TIOLINES: TiO now, VO, $\mathrm{ZrO}$, etc, in the future;

TRIATOMICS: empty now, $\mathrm{H}_{2} \mathrm{O}$, etc., in the future.

For each line the wavelength, identification, lower energy level, $g f$ value, and the radiative, Stark, and van der Waals damping constants are packed into 16 bytes. At each wavelength point in a frequency integration, the Voigt profiles of all the significant nearby lines are computed and summed. The lines in file NLTELINES can be treated with more complicated profile functions. These files are available on Kurucz CD-ROMs Nos. 1 and 15. ATLAS12 is still not working reliably at this writing. I hope to distribute it in the fall of 1993 . It has been 
used to make models with 25000 sampled frequencies for Vega (Kurucz 1993; Castelli \& Kurucz 1993), which is an easy case.

It will be possible to use ATLAS12 to compute Rosseland opacities for arbitrary abundances for use in envelope and interior calculations.

\section{Complete Spectrum Synthesis}

It is now possible simply to compute the whole spectrum using all 58 million lines without resorting to sampling or statistical approximations. Such a calculation takes a few days on a very fast workstation. Figure 1, on the six pages following Table 2, shows a sample calculation for Arcturus using an improved version of the model atmosphere from Peterson et al. (1993) that was computed with ATLAS9. The spectrum calculation shown in the top panel of Fig. 1a,1c,1e was repeated 11 times with subsets of the line list. The resolution was 500,000 with $1,864,857$ points between 200 and $10000 \mathrm{~nm}$. A full dispersion plot that displays the resolution requires a case of paper. It is now also possible to compute models by integrating over the whole spectrum for each iteration, but even I am daunted by this prospect, for the moment.

\section{References}

Anders E., Grevesse N., 1989, Geochimica et Cosmochimica Acta, 53, 197

Avrett E.H., Kurucz R.L., Loeser R., 1984, Bull. Amer. Astron. Soc., 16, 450

Avrett E.H., Loeser R., 1992, in 7th Cambridge Workshop on Cool Stars, Stellar Systems, and the Sun, M.S.Giampapa \& J.A.Bookbinder (eds.), ASP Conference Series, 26, 489

Castelli F., Kurucz R.L., 1993, Astron. Astrophys., in press

Cowan R.D., 1968, J. Opt. Soc. Am.58, 808

Farrenq R., Guelachvili G., Sauval A.J., Grevesse N., Farmer C.B., 1991, J. Mol. Spectr., 149, 375

Jørgensen U.G., Jensen P., 1993, J. Mol. Spectr., 161, 219

Kurucz R.L., 1970, SAO Spec. Rep.No. 309, 291 pp.

Kurucz R.L., 1979a, Astrophys. J. Supp., 40, 1

Kurucz R.L., 1979b, Dudley Observ. Rep. No. 14, A.G.Davis Philip (ed.), p. 363

Kurucz R.L., 1992a, Rev. Mexicana Astron. Astrof., 23, 45

Kurucz R.L., 1992b, Rev. Mexicana Astron. Astrof., 23, 181

Kurucz R.L., 1993a, in Peculiar versus Normal Phenomena in A-type and Related Stars, M.M.Dworetsky, F.Castelli, \& R.Faraggiana (eds.), ASP Conference Series, 44, 87

Kurucz R.L., 1993b, Physica Scripta, T47, 110

Kurucz R.L., Avrett E.H., 1981, SAO Spec. Rep.No. 391, 145 pp.

Kurucz R.L., Peytremann, E., 1975, SAO Spec. Rep.No. 362, 1219 pp.

Peterson D.M., 1979, Personal communication

Peterson R.C., dalle Ore C.M., Kurucz R.L., 1993, Astrophys. J., 404, 303

Vidal C.R., Cooper J., Smith E.W., 1973, Astrophys. J. Supp., 25, 37 


\section{Table 2. Kurucz CD-ROMs}

Available from the author (Kurucz@cfa.harvard.edu)

No.01 Atomic data for opacity calculations.

No.02 Opacities for Stellar Atmospheres: $[+0.0],[+0.5],[+1.0]$.

No.03 Opacities for Stellar Atmospheres: $[-0.5],[-1.0],[-1.5]$.

No.04 Opacities for Stellar Atmospheres: $[-2.0],[-2.5],[-3.0]$.

No.05 Opacities for Stellar Atmospheres: $[+0.1],[+0.2],[+0.3]$.

No.06 Opacities for Stellar Atmospheres: $[-0.1],[-0.2],[-0.3]$.

No.07 Opacities for Stellar Atmospheres: [-3.5],[-4.0],[-4.5].

No.08 Opacities for Stellar Atmospheres: [-5.0], $[+0.0$, noHe], $[-0.5$, noHe].

No.09 Opacities for Stellar Atmospheres: [+0.5a],[+0.0a],[-0.5a] +.4 alpha.

No.10 Opacities for Stellar Atmospheres: $[-1.0 \mathrm{a}],[-1.5 \mathrm{a}],[-2.0 \mathrm{a}]+.4$ alpha.

No.11 Opacities for Stellar Atmospheres: $[-2.5 \mathrm{a}],[-3.0 \mathrm{a}],[-3.5 \mathrm{a}]+.4$ alpha.

No.12 Opacities for Stellar Atmospheres: [-4.0a], $[-4.5 \mathrm{a}],[-5.0 \mathrm{a}]+.4$ alpha.

No.13 ATLAS9 Stellar Atmosphere Programs and $2 \mathrm{~km} / \mathrm{s}$ Grid.

No.14 Opacities for Stellar Atmospheres: Abundance Sampler.

No.15 Diatomic molecular data for opacity calculations.

In preparation:

Replacement for Kurucz-Peytremann atomic line list for all elements.

Molecular line list with all the old data (Table I).

ATLAS12 opacity sampling model atmosphere program and

SYNTHE spectrum synthesis programs.

Model Atmosphere Grids: [+0.0] for $0,1,2,4,8 \mathrm{~km} / \mathrm{s}$ Vturb.

Model Atmosphere Grids: [-0.5a], $[+0.0 \mathrm{a}],[+0.5 \mathrm{a}] 2 \mathrm{~km} / \mathrm{s}$ for bulge stars.

Model Atmosphere Grids: Limbdarkening for $2 \mathrm{~km} / \mathrm{s}$ Grid (No.13).

\section{Coming:}

All new atomic and molecular calculations as they are made.

Improved atomic data for opacity calculations.

Improved molecular data for opacity calculations.

Model Atmosphere Grids: [-1.0a], $[-1.5 \mathrm{a}],[-2.0 \mathrm{a}] 2 \mathrm{~km} / \mathrm{s}$.

Model Atmosphere Grids: [-2.5a],[-3.0a],[-3.5a] $2 \mathrm{~km} / \mathrm{s}$.

Model Atmosphere Grids: [-4.0a],[-4.5a],[-5.0a] $2 \mathrm{~km} / \mathrm{s}$.

Model Atmosphere Grids: [-0.5] for $0,1,2,4,8 \mathrm{~km} / \mathrm{s}$ Vturb.

Model Atmosphere Grids: [-1.0] for $0,1,2,4,8 \mathrm{~km} / \mathrm{s}$ Vturb.

Model Atmosphere Grids: $[-1.5]$ for $0,1,2,4,8 \mathrm{~km} / \mathrm{s}$ Vturb.

Model Atmosphere Grids: [-2.0] for $0,1,2,4,8 \mathrm{~km} / \mathrm{s}$ Vturb.

Model Atmosphere Grids: [+0.5] for 0,1,2,4,8 km/s Vturb.

Model Atmosphere Grids: [+0.0a] for $0,1,2,4,8 \mathrm{~km} / \mathrm{s}$ Vturb.

Model Atmosphere Grids: [-0.5a] for $0,1,2,4,8 \mathrm{~km} / \mathrm{s}$ Vturb.

Model Atmosphere Grids: [-1.0a] for $0,1,2,4,8 \mathrm{~km} / \mathrm{s}$ Vturb.

Model Atmosphere Grids: [-1.5a] for $0,1,2,4,8 \mathrm{~km} / \mathrm{s}$ Vturb.

Model Atmosphere Grids: [-2.0a] for $0,1,2,4,8 \mathrm{~km} / \mathrm{s}$ Vturb.

Model Atmosphere Grids: [+0.5a] for $0,1,2,4,8 \mathrm{~km} / \mathrm{s}$ Vturb. 


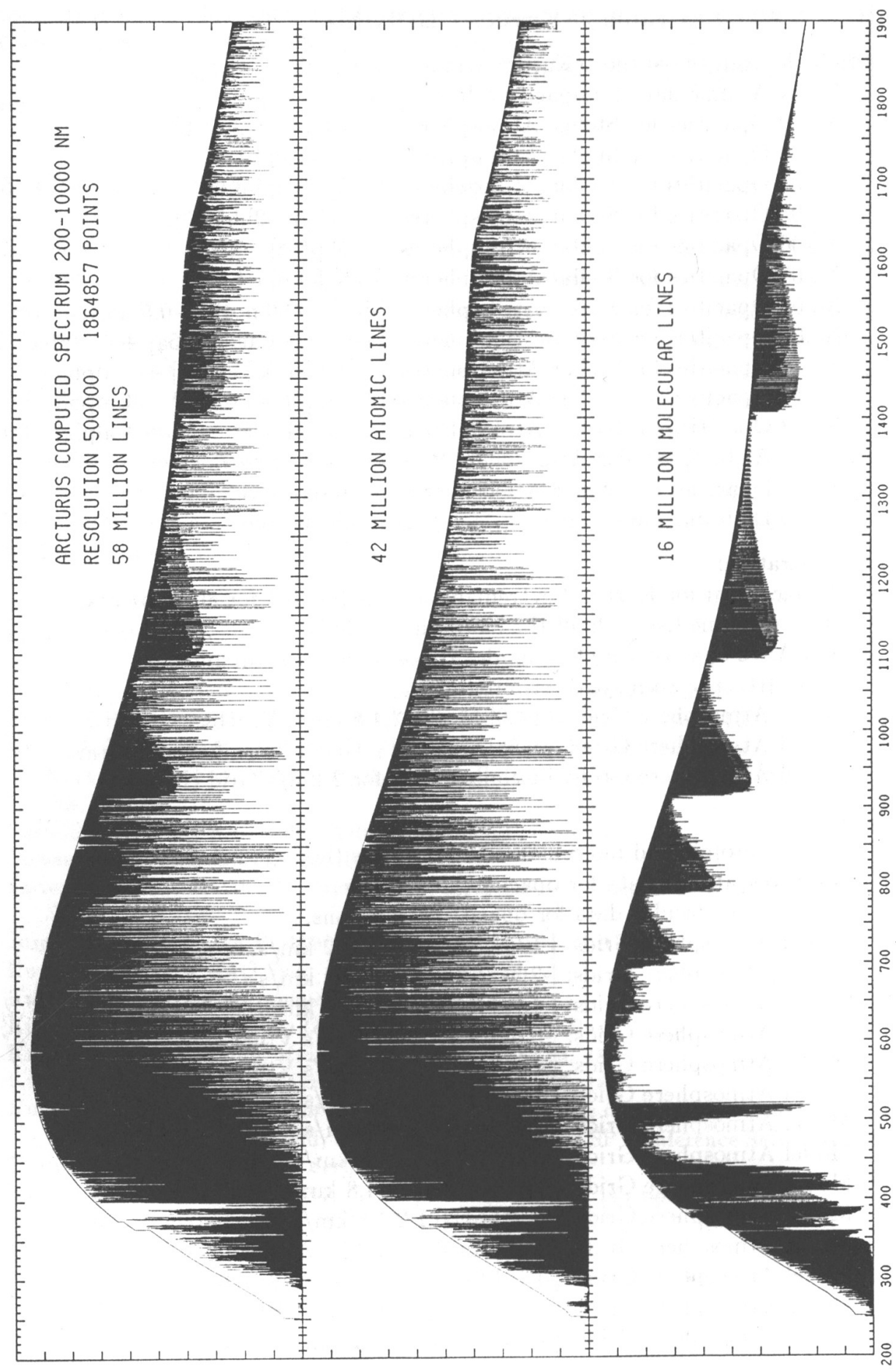




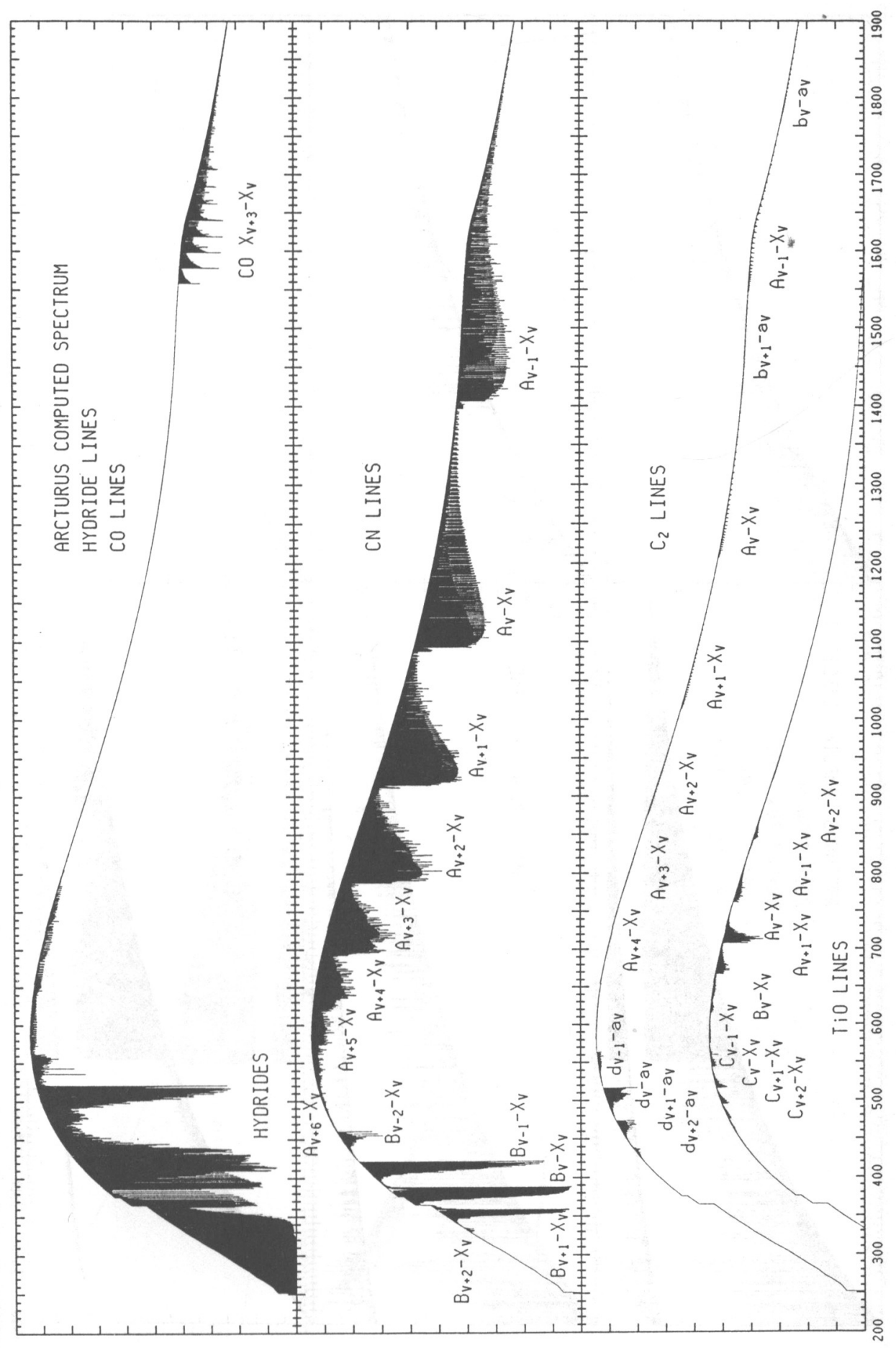




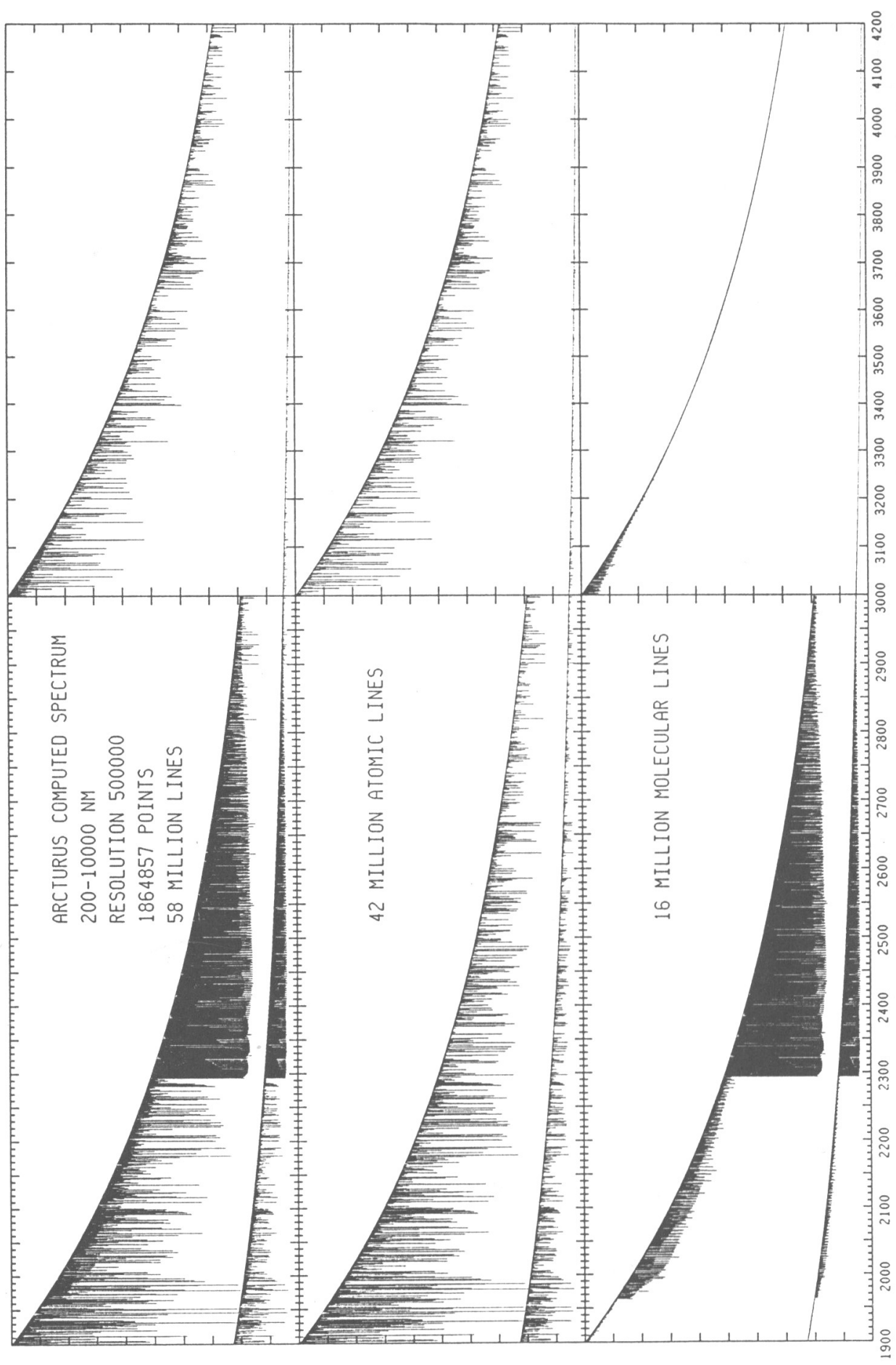




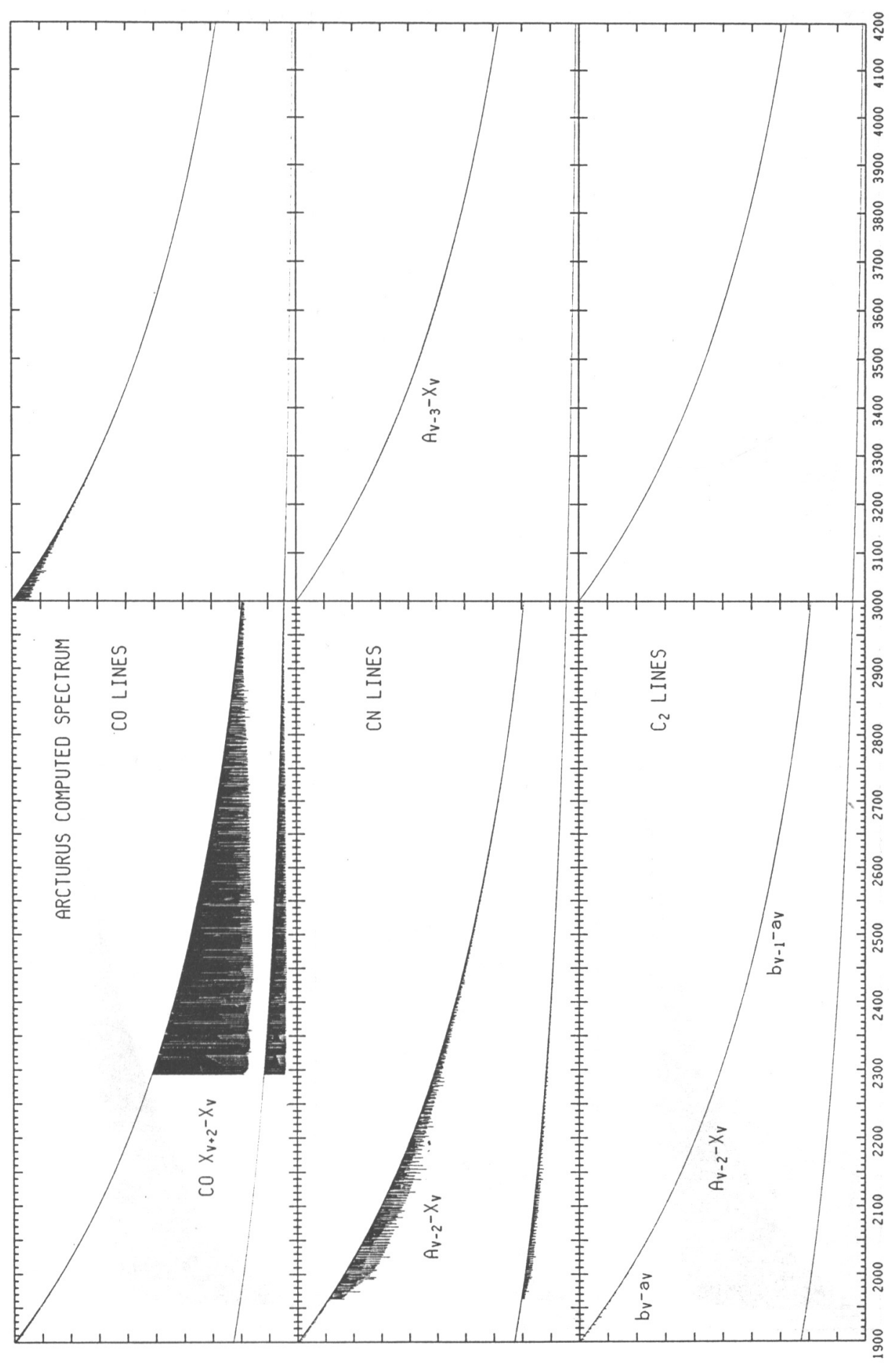




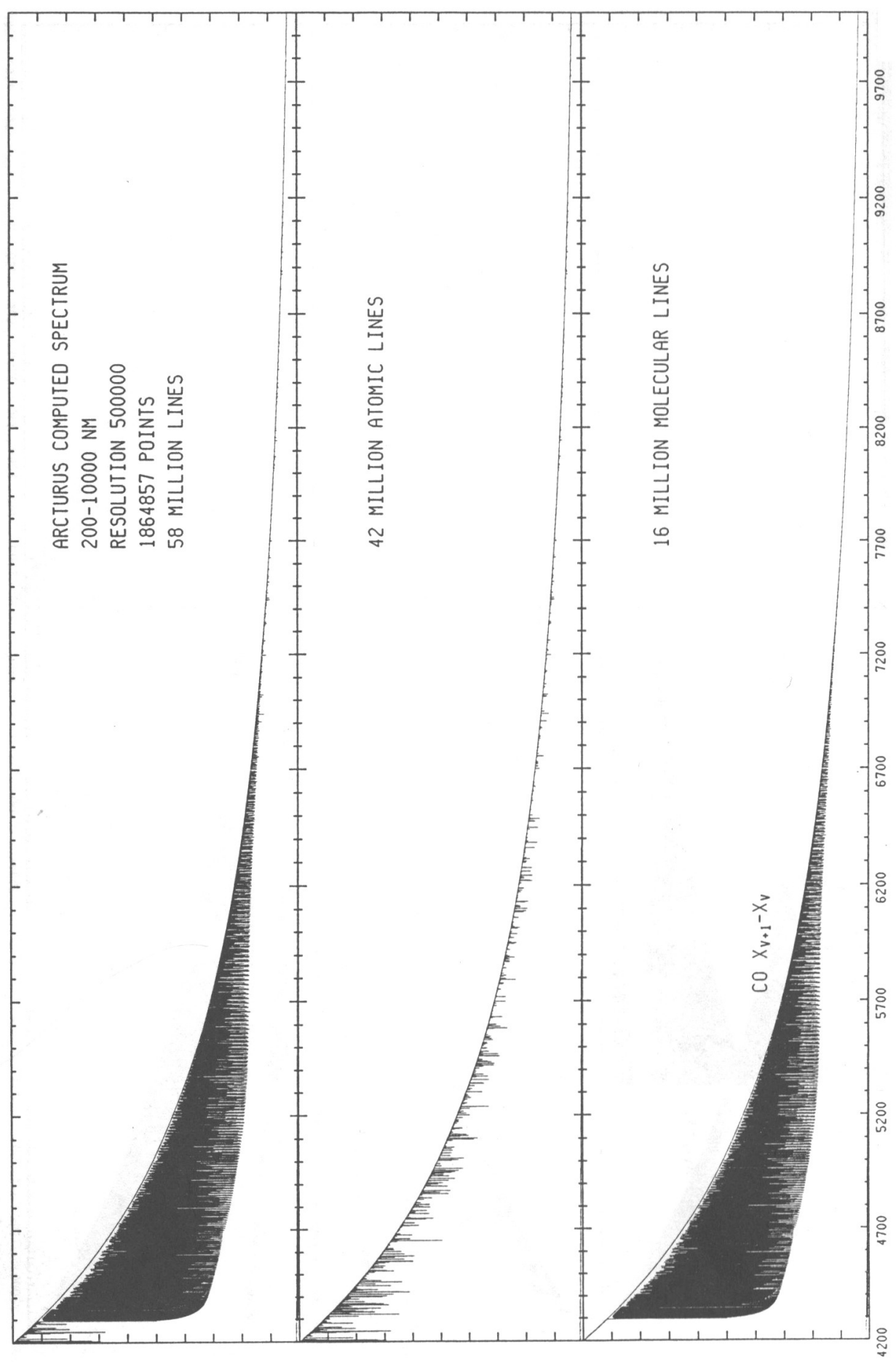




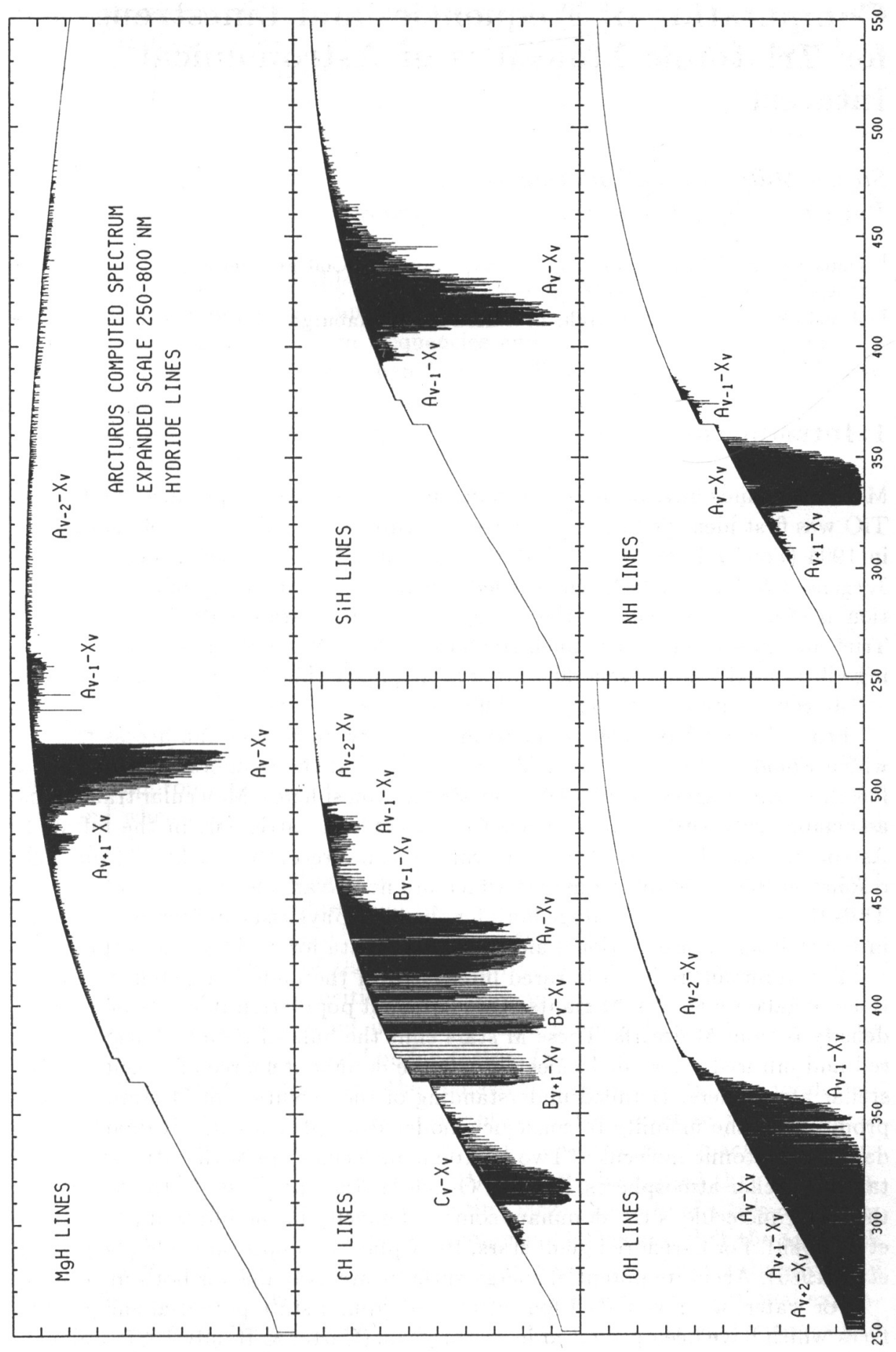

